\title{
A98-37125
}

\section{STABILITY ANALYSIS OF DYNAMIC INVERSION CONTROLLERS USING TIME-SCALE SEPARATION}

\author{
Corey J. Schumacher* Pramod P. Khargonekar ${ }^{\dagger} \quad$ N. Harris McClamroch ${ }^{\ddagger}$
}

\begin{abstract}
ASTRACT
This paper examines the closed-loop stability of a nonlinear system using a two time-scale dynamic inversion controller. A state-space formulation for the system is derived, assuming the inner-loop inversion is performed exactly. A Lyapunov analysis is then performed to show that, under certain assumptions, the exponential stability of the system about constant commanded state values is guaranteed with a sufficiently large inner-loop gain. For a given gain, a method is given to estimate the domain of attraction of the equilibrium about the commanded state values. The primary advantage of this method over results obtained via singular perturbation analysis is that it provides useful estimates of the domain of attraction, as well as a sufficient gain to guarantee stability.
\end{abstract}

\section{INTRODUCTION}

- Research Aerospace Engineer, Air Force Research Laboratory, Air Vehicles Directorate, AFRL/VACC, Bldg. 146, 2210 Eighth St., Suite 21, Wright-Patterson Air Force Base, OH 45433-7531. Tel. (937) 255-8678, Fax (937) 656-4000. Email. coreys@falcon.fight.wpafb.af.mil

tDepartment of Electrical Engineering and Computer Science, The University of Michigan, 1301 Beal Ave, Ann Arbor, MI 48109-2122, USA. Tel (313) 764-4328, Fax (313) 763-8041, Email: pramod@eecs.umich.edu

¿Department of Aerospace Engineering, The University of Michigan, 1320 Beal Ave, Ann Arbor, wil 48109-2118, USA. Tel (313) 763-2355, Fax (313) 763-0578, Email: nhm@engin.umich.edu

${ }^{1}$ This paper is declared a work of the U.S. government and is not subject to copyright protection in the United States
Time-scale separation exists in many dynamical systems. Time-scale separations can arise due to small time constants, moments of inertia, flexible body dynamics, actuator dynamics, and many other effects. Using the natural separation between "fast" and "slow" variables to reduce the complexity of a dynamical system can greatly simplify the control design and analysis problem.

There are natural time-scale separations in many flight control problems, both in the design of attitudecontrol autopilots and trajectory-optimization problems. Examples of dynamic inversion flight control using time-scale separation can be found in $[7,8,9,5,11]$.

Typically, the effect of time-scale separation in a dynamical system is studied using singular perturbation theory. In this method, the "fast" dynamics are assumed to go to steady state, and the stability of the resulting, simplified system is studied. Singular perturbation theory is well developed, and has been applied to many different control problems that exhibit a time-scale separation. Detailed discussions of singular perturbation theory can be found in $[1,2,3,4]$. A detailed discussion of the use of singular perturbations and time scales in aerospace systems can be found in [6]. This paper contains an extensive list of references for the use of singular perturbation theory in aerospace systems.

In this paper, the stability of a nonlinear system with a two time-scale structure with a dynamic inver- 
sion controller is examined. The system is of a form where the fast variables can be used as control inputs for the slow variables. Systems of this form appear frequently in aerospace applications. Two dynamic inversion controllers are used, an outer-loop inversion using the fast states as controls for the slow states, and an inner-loop inversion using the control inputs to control the fast states.

This paper presents a Lyapunov stability analysis of the closed-loop system formed by the nonlinear system and the dynamic inversion controllers. The main result of the paper is stated as Theorem 0.1. The proof of this theorem takes up the majority of the paper. By assuming the fast inversion is performed exactly, the closed-loop system can be converted into a second-order form in the slow variables. The stability of this state-space system is then analyzed. It is proven that the system is exponentially stable about constant, commanded values of the outer-loop states for a sufficiently large inner-loop gain. A sufficient condition for stability, and a domain of attraction for the commanded states are calculated. A detailed application of this type of stability analysis to an airto-air missile autopilot design problem can be found in [7] and [10].

\section{PROBLEM FORMULATION}

Suppose a nonlinear system is of the form

$$
\begin{aligned}
& \dot{x}=f(x)+g(x) y \\
& \dot{y}=h(x, y)+k(x, y) u
\end{aligned}
$$

where $x$ are slow states, $y$ are fast states, and $u$ is the control. The vectors $x, y$, and $u$ are all $n$-dimensional and real-valued. In this system, $\dot{x}$ is affine in $y$, and $\dot{y}$ is affine in $u$. This is similar to the system used in dynamic inversion control of an aircraft using a two time-scale separation. Further suppose the following assumptions hold:

- Assumption 1. The functions $g(x)$ and $k(x, y)$ are invertible.
- Assumption 2. The functions $f(x), g(x)$, $h(x, y)$, and $k(x, y)$ are finite inside a level set of a Lyapunov function $V$ for the system which will be defined shortly.

- Assumption 3. The derivatives of $f(x)$ and $g(x)$ with respect to $x$ are finite inside the level set of $V$.

- Assumption 4. The desired value of $x, x=x_{c}$, is constant.

The two time-scale dynamic inversion controller for this system is of the form:

$$
\begin{aligned}
u & =k^{-1}(x, y)\left(\dot{y}_{d}-h(x, y)\right) \\
y_{c} & =g^{-1}(x)\left(\dot{x}_{d}-f(x)\right)
\end{aligned}
$$

with

$$
\begin{aligned}
& \dot{x}_{d}=\Omega\left(x_{c}-x\right) \\
& \dot{y}_{d}=\omega_{i} I\left(y_{c}-y\right)
\end{aligned}
$$

and

$$
\Omega=\left[\begin{array}{ccc}
\omega_{O 1} & & 0 \\
& \ddots & \\
0 & & \omega_{o n}
\end{array}\right] .
$$

The result of the generalized stability analysis can now be stated as Theorem 0.1 .

Theorem 0.1 Suppose Assumptions 1-4 hold for the dynamical system given by Equations 1-2. Then, with the dynamic inversion controller specified by Equations $3-6$, the states $x$ will be exponentially stable about their commanded values for any gain $\omega_{i} \geq \omega_{i}^{*}$. This $\omega_{i}^{*}$ can be found from Equation 31.

Proof: The proof is presented in several steps, taking up the balance of the paper. 


\section{STABILITY ANALYSIS}

The first step of the proof is to convert the dynamical system into a state-space system for $x$ and $\dot{x}$ only. The stability of this system will then be studied using Lyapunov analysis.

\section{Derivation of State-Space System}

First, rewrite $g(x)$ as

$$
g(x)=\left[g_{1}(x), g_{2}(x), \ldots, g_{n}(x)\right],
$$

where $g_{i}(x)$, is an $n \times 1$ vector function. Then, taking the derivative of Equation 1 with respect to time gives $\ddot{x}=\frac{\partial f}{\partial x} \dot{x}+\left[\frac{\partial g_{1}}{\partial x} \dot{x}, \frac{\partial g_{2}}{\partial x} \dot{x}, \ldots, \frac{\partial g_{n}}{\partial x} \dot{x}\right] y+g(x) \dot{y}$.

Substituting $y=g^{-1}(x)(\dot{x}-f(x)), \quad y_{c}=$ $g^{-1}(x)\left(\dot{x}_{d}-f(x)\right)$, and $\dot{y}=\omega_{i} I\left(y_{c}-y\right)$ into Equation 8 gives:

$$
\begin{aligned}
\ddot{x}= & \frac{\partial f}{\partial x} \dot{x}+\left[\frac{\partial g_{1}}{\partial x} \dot{x}, \frac{\partial g_{2}}{\partial x} \dot{x}, \ldots, \frac{\partial g_{n}}{\partial x} \dot{x}\right] \\
& \cdot g^{-1}(x)(\dot{x}-f(x))+g(x) \omega_{i} I\left[g^{-1}(x)\right. \\
& \left.\cdot\left(\dot{x}_{d}-f(x)\right)-g^{-1}(x)(\dot{x}-f(x))\right]
\end{aligned}
$$

which simplifies to

$$
\begin{aligned}
\ddot{x}= & \frac{\partial f}{\partial x} \dot{x}+\left[\frac{\partial g_{1}}{\partial x} \dot{x}, \frac{\partial g_{2}}{\partial x} \dot{x}_{,} \ldots, \frac{\partial g_{n}}{\partial x} \dot{x}\right] \\
& \cdot g^{-1}(x)(\dot{x}-f(x))+\omega_{i} I\left[\dot{x}_{d}-\dot{x}\right] .
\end{aligned}
$$

Substituting for $\dot{x}_{d}$ and rearranging results in:

$$
\begin{aligned}
& \ddot{x}-\left(\frac{\partial f}{\partial x}-\omega_{i} I\right) \dot{x}+\omega_{i} \Omega\left(x-x_{c}\right) \\
& -\left[\frac{\partial g_{1}}{\partial x} \dot{x}, \frac{\partial g_{2}}{\partial x} \dot{x}, \ldots, \frac{\partial g_{n}}{\partial x} \dot{x}\right] g^{-1}(x)(\dot{x}-f(x))=0 .
\end{aligned}
$$

Now, define $z_{1}=x-x_{c}$. For constant $x_{c}, \dot{z}_{1}=\dot{x}$. Then define $z_{2}=\dot{x}$. A state space system for $z=$ $\left(z_{1}^{T}, z_{2}^{T}\right)^{T}$ is given by:

$$
\begin{aligned}
\dot{z}_{1}= & z_{2} \\
\dot{z}_{2}= & -\omega_{i} \Omega z_{1}+\left(\frac{\partial f}{\partial x}-\omega_{i} I\right) z_{2} \\
& +\left[\frac{\partial g_{1}}{\partial x} z_{2}, \frac{\partial g_{2}}{\partial x} z_{2}, \ldots, \frac{\partial g_{n}}{\partial x} z_{2}\right] \\
& \cdot g^{-1}(x)\left(z_{2}-f(x)\right) .
\end{aligned}
$$

The stability of this nonlinear state-space system will now be examined.

\section{Lyapunov Stability Analysis}

The first step of the stability analysis is to examine the equilibria of Equations 12. It is clear from inspection that $z_{1}=0, z_{2}=0$ is the only equilibrium of the system.

Let $V=\frac{1}{2} z^{T} P z$ be a Lyapunov function candidate for the system (12) with

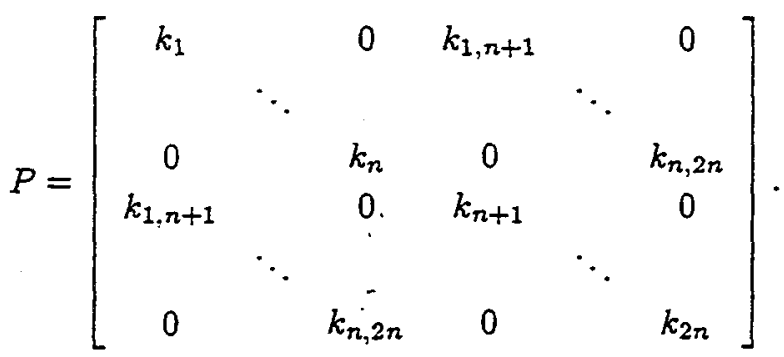

where $k_{i}, k_{i, j} \in \Re$ must be chosen to make $P$ positive definite. They must also all be greater than zero. The matrix $P$ can be rewritten as four submatrices:

$$
P=\left[\begin{array}{ll}
P_{11} & P_{12} \\
P_{12} & P_{22}
\end{array}\right]
$$

with the obvious definitions of $P_{11}, P_{12}$, and $P_{22}$. Note that the $P_{i j}$ 's are diagonal, real positive defnite matrices.

Taking the derivative of $V$ along (12) gives

$$
2 \dot{V}=\dot{z}^{T} P z+z^{T} P \dot{z}
$$

which, with the substitution $\dot{z}_{1}=z_{2}$, expands out to:

$$
\begin{aligned}
2 \dot{V}= & z_{2}^{T} P_{11} z_{1}+z_{2}^{T} P_{12} z_{2}+\dot{z}_{2}^{T} P_{12} z_{1} \\
& +\dot{z}_{2}^{T} P_{22} z_{2}+z_{1}^{T} P_{11} z_{2}+z_{1}^{T} P_{12} \dot{z}_{2} \\
& +z_{2}^{T} P_{12} z_{2}+z_{2}^{T} P_{22} \dot{z}_{2} .
\end{aligned}
$$

Note that $\dot{z}_{2}^{T} P_{12} z_{1}=z_{1}^{T} P_{12} \dot{z}_{2}$, and similarly for the other terms. This allows all of the derivatives in Equation 13 to be moved to the right sides of the matrices. Then

$\dot{V}=z_{2}^{T} P_{11} z_{1}+z_{2}^{T} P_{12} z_{2}+z_{1}^{T} P_{12} \dot{z}_{2}+z_{2}^{T} P_{22} \dot{z}_{2}$. 
Let $\ddot{z}_{2}$ from Equation 12 be written as

$$
\dot{z}_{2}=-\omega_{i} I z_{2}-\omega_{i} \Omega z_{1}+l(z)
$$

with

$$
\begin{aligned}
l(z)= & \frac{\partial f}{\partial x} z_{2}+\left[\frac{\partial g_{1}}{\partial x} z_{2}, \frac{\partial g_{2}}{\partial x} z_{2}, \ldots, \frac{\partial g_{\pi}}{\partial x} z_{2}\right] \\
& \cdot g^{-1}(x)\left(z_{2}-f(x)\right) .
\end{aligned}
$$

Then, with this substitution for $\dot{z}_{2}, \dot{V}$ can be written as

$$
\begin{aligned}
\dot{V}= & -\omega_{i} z_{1}^{T} P_{12} \Omega z_{1}+z_{2}^{T}\left(P_{12}-\omega_{i} P_{22}\right) z_{2} \\
& +z_{2}^{T}\left(P_{11}-\omega_{i} P_{12}-\omega_{i} P_{22} \Omega\right) z_{1} \\
& z_{1}^{T} P_{12} l(z)+z_{2}^{T} \omega_{i} P_{22} l(z) .
\end{aligned}
$$

The only requirement on $P$ is that it be positive definite. Therefore, freedom exists in the choice of the constants $k_{i}, k_{i j}$. Therefore, let the $k_{i}, k_{i j}$ 's be chosen so that

$$
P_{11}=\omega_{i} P_{12}+-\omega_{i} P_{22} \Omega .
$$

With this choice of $P_{11}$, the $z_{2}^{T}\left(P_{11}-\omega_{i} P_{12}-\omega_{i} P_{22} \Omega\right) z_{1}$ term will drop out of $\dot{V}$. Then

$$
\begin{aligned}
\dot{V}= & -\omega_{i} z_{1}^{T} P_{12} \Omega z_{1}+z_{2}^{T}\left(P_{12}-\omega_{i} P_{22}\right) z_{2} \\
& +z_{1}^{T} P_{12} l(z)+z_{2}^{T} P_{22} l(z) .
\end{aligned}
$$

The first two terms of $\dot{V}$ in Equation 20 form, for a large enough $\omega_{i}$, a negative definite quadratic form in $z$. If the magnitude of this function is larger than the magnitude of the rest of the terms on the right-hand side of Equation 20, then $\dot{V}$ is negative definite. The next step must be to examine these terms.

\section{Analysis of $\dot{V}$}

Recalling Equation 16, the problematic terms in $\dot{V}$ are

$$
\begin{aligned}
& l(z)=\frac{\partial f}{\partial x} z_{2}+\left[\frac{\partial g_{1}}{\partial x} z_{2}, \frac{\partial g_{2}}{\partial x} z_{2}, \ldots, \frac{\partial g_{n}}{\partial x} z_{2}\right] \\
& \cdot g^{-1}(x)\left(z_{2}-f(x)\right) .
\end{aligned}
$$

The expression for $l(z)$ can be broken up into three elements, which will be discussed separately:
1. $\frac{\partial f}{\partial x} z_{2}$,

2. $\left[\frac{\partial g_{1}}{\partial x} z_{2}, \frac{\partial g_{2}}{\partial x} z_{2}, \ldots, \frac{\partial g_{n}}{\partial x} z_{2}\right] g^{-1}(x) z_{2}$, and

3. $-\left[\frac{\partial g_{1}}{\partial x} z_{2}, \frac{\partial g_{2}}{\partial x} z_{2}, \ldots, \frac{\partial g_{n}}{\partial x} z_{2}\right] g^{-1}(x) f(x)$.

Element 1: This element will create terms in $\dot{V}$ of the form

$$
z_{1}^{T} P_{12} \frac{\partial f}{\partial x} z_{2}+z_{2}^{T} P_{22} \frac{\partial f}{\partial x} z_{2} .
$$

Element 2: This element of $l(z)$ is more complex. The term $\frac{\partial g}{\partial x}$ is the derivative of a matrix with respect to a vector. Multiplying it by $z_{2}$ results in a matrix, every non-zero term of which is multiplied by an element of $z_{2}$. This matrix is then multiplied by $g^{-1}(x) z_{2}$, resulting in a vector with every nonzero term being quadratic in elements of $z_{2}$. This will create terms in $\dot{V}$ of the cubic in elements of $z$, all of which are at least quadratic in elements of $z_{2}$

Element 3: This element of $l(z)$ is a vector, all of whose non-zero elements are linear in elements of $z_{2}$. This will create some terms in $\dot{V}$ which are quadratic in elements of $z_{1}$ and $z_{2}$, and some terms which are quadratic in elements of $z_{2}$.

$$
\text { So, } \begin{aligned}
z_{1}^{T} P_{12} l(z) & +z_{2}^{T} P_{22} l(z)=m(z), \text { where } \\
m(z)= & \sum_{i=1}^{n} \sum_{j=n+1}^{2 n}\left(z_{i} z_{j} a_{i j}+z_{i} z_{j}^{2} b_{i j}\right) \\
& +\sum_{i=n+1}^{2 n} \sum_{j=n+1}^{2 n} z_{i} z_{j} c_{i j} \\
& +\sum_{i=n+1}^{2 n} \sum_{j=n+1}^{2 n} \sum_{k=n+1}^{2 n} z_{i} z_{j} z_{k} d_{i j k}
\end{aligned}
$$

These coefficients are composed of terms from $P_{12}$, $P_{22}, \frac{\partial f}{\partial x}, \frac{\partial g}{\partial x}, g^{-1}(x)$, and $f(x)$.

Rewriting Equation 20 using $m(z)$ gives:

$\dot{V}=-\omega_{i} z_{1}^{T} P_{12} \Omega z_{1}+z_{2}^{T}\left(P_{12}-\omega_{i} P_{22}\right) z_{2}+m(z) .(22)$

Therefore

$\dot{V} \leq-\omega_{i} z_{1}^{T} P_{12} \Omega z_{1}+z_{2}^{T}\left(P_{12}-\omega_{i} P_{22}\right) z_{2}+|m(z)| \cdot(23)$ 
From this development, it is clear that $l(z)$ causes terms cubic in $z$ to appear in $\dot{V}$. For $\dot{V}$ to be negative definite, these terms must be bounded in some manner.

\section{Bounds on the Elements of $l(z)$}

The size of the elements of $z$ can be bounded by examining a level set of $V$. When a command in $x$ is given, this creates an inital condition $z_{0}$ in $z$, and the goal of the system is then to drive $z$ to the origin. This initial condition $z_{0}$ has an associated initial value $V_{0}$ for the Lyapunov function $V$. Let $z_{10}$ be the initial condition for the first element of $z$ and define

$$
V_{0}=\frac{1}{2} k_{1} z_{10}^{2}
$$

and note that $V_{0}$ defines a level set $S_{0}$ of $V$. If $\dot{V}$ is negative definite inside $S_{0}$, then the system trajectory will never leave this level set.

Let $\mathcal{D}$ be the smallest hyperbox that contains the level set $S_{0}$. The bounds on the states in $\mathcal{D}$ can be calculated from $V_{0}$. Note that the form of $P$ is such that the $z_{i}$ and $z_{i+n}$ elements are decoupled from the rest of $z$ in the calculation of $V$. Since $V$ is positive definite, the largest values of $z_{i}$ and $z_{n+i}$ in the level set $S_{0}$ occur when all other elements of $z$ are zero. The maximal value for $z_{i+n}$ can be found by differentiating the equation

$$
V=\frac{1}{2} k_{i} z_{i}^{2}+\frac{1}{2} k_{n+i} z_{n+i}^{2}+k_{i, n+i} z_{i} z_{n+i}
$$

with respect to $z_{i}$ and finding

$$
\frac{\partial x_{2}}{\partial x_{1}}=-\frac{k_{1} x_{1}+k_{5} x_{2}}{k_{2} x_{2}+k_{5} x_{1}}
$$

along the level set of $V$. Setting $\frac{\partial z_{i+n}}{\partial z_{i}}=0$ gives $z_{i+n}=-\frac{k_{i}}{k_{i, n+1}} z_{i}$. Substituting back into Equation 24 , results in

$$
z_{i}=\sqrt{\frac{2 V_{10} k_{i, n+1}^{2}}{k_{n}+i k_{i}^{2}-k_{i} k_{i, n+i}^{2}}}
$$

Substituting this equation into $z_{i+n}=-\frac{k_{i}}{k_{i, n+1}} z_{i}$ gives the maximum value for $z_{i+n}$ on the level set $S_{0}$ :

$$
\left|z_{i+n}\right| \leq \sqrt{\frac{2 V_{0} k_{i}}{k_{i} k_{i+n}-k_{i, n+i}^{2}}}
$$

Substituting $V_{0}=\frac{1}{2} k_{1} z_{10}^{2}$ and $k_{1}=k_{1+n} \omega_{i} \omega_{01}+$ $k_{1,1+n} \omega_{i}$ into Equation 25 and simplifying gives

$$
\begin{gathered}
\left|z_{i+n}\right| \leq\left|z_{10}\right| \sqrt{\omega_{i}} \sqrt{\frac{k_{1+n} \omega_{o 1}+k_{1,1+n}}{k_{i+n}}} \\
\cdot \sqrt{\frac{1}{1-\frac{k_{i, i+n}^{2}}{k_{i} k_{i+n}}}}
\end{gathered}
$$

Finally, if $\omega_{i}>1$, then

$$
\begin{aligned}
\left|z_{i+n}\right| \leq & \sqrt{\frac{1}{1-\frac{k_{i, i+n}^{2}}{k_{i+n}\left(k_{i+n} \omega_{a i}+k_{i, i+n}\right)}}} \\
& \sqrt{\frac{k_{1+n} \omega_{01}+k_{1,1+n}}{k_{i+n}}}\left|z_{1_{0}}\right| \sqrt{\omega_{i}}
\end{aligned}
$$

The maximum value of $z_{i+n}$ in $S_{0}$ is proportional to $\sqrt{\omega_{i}}$ and can be rewritten as $\left|z_{i+n}\right| \leq K_{i+n} \sqrt{\omega_{i}}$.

A similar development can be done for $z_{i}, i=$ $1, \ldots, n$, resulting in

$$
\begin{aligned}
\left|z_{i}\right|= & \leq \sqrt{\frac{1}{1-\frac{k_{i, i+n}^{2}}{k_{i+n}\left(k_{i+n} \omega_{0 i}+k_{i, i+n}\right)}}} \\
& \cdot \sqrt{\frac{k_{1+n} \omega_{o 1}+k_{1,1+n}}{k_{i+n} \omega_{o i}+k_{i, i+n}}\left|z_{10}\right|}
\end{aligned}
$$

The maximum value of $z_{i}$ in $S_{0}$ can be bounded by a constant $K_{i}$, irrespective of $\omega_{i}$. Without the simplification for $\omega_{i} \geq 1$, it would in fact decrease slightly with increasing $\omega_{i}$. So, $\left|z_{i}\right| \leq K_{i}$ in $S_{0}$.

Let $\mathcal{D}$ be the hyperbox defined by Equations 26 and 27. The set $\mathcal{D}$ contains the level set $S_{0}$. If $\dot{V}$ is negative definite (or negative semidefinite) in $S_{0}$, the system trajectory will never leave $S_{0}$, and therefore will never leave $\mathcal{D}$. By Assumptions $A 11$ and $A 12$, bounds on the magnitudes of the individual terms of 
$\frac{\partial f}{\partial x}, \frac{\partial g}{\partial x}, g^{-1}(x)$, and $f(x)$ can be computed inside of $\mathcal{D}$. These bounds also will never be exceeded if $\dot{V}$ is negative definite in $S_{0}$.

\section{Continued Analysis of $\dot{V}$}

Each of the terms in $m(z)$ can now be bounded inside $\mathcal{D}$. The coefficients of the quadratic terms can be bounded using the bounds on $z_{1}$ and $\frac{\partial f}{\partial x}, \frac{\partial g}{\partial x}$, $g^{-1}(x)$, and $f(x)$. Using the bounds on the states given in Equations 26, 27, the cubic terms in $m(z)$ from Equation 21 can be reduced to second order in $z$. The cubic terms are of the form

$$
\sum_{i=n+1}^{2 n} \sum_{j=n+1}^{2 n} \sum_{k=n+1}^{2 n} z_{i} z_{j} z_{k} d_{i j k}
$$

and

$$
\sum_{i=1}^{n} \sum_{j=n+1}^{2 n} z_{i} z_{j}^{2} b_{i j}
$$

Using Equation 26, these terms can be individually bounded by:

$$
\left|z_{i} z_{j} z_{k} d_{i j k}\right| \leq\left|z_{i} z_{j} K_{k} d_{i j k} \sqrt{\omega_{i}}\right|
$$

and

$$
\left|z_{i} z_{j}^{2} b_{i j}\right| \leq\left|K_{i} z_{j}^{2} b_{i j}\right|
$$

Therefore,

$$
\left|z_{i} z_{j} z_{k} d_{i j k}\right| \leq\left|z_{i} z_{j} e_{i j} \sqrt{\omega_{i}}\right|
$$

for some real constants $e_{i j}$ and

$$
\left|z_{i} z_{j}^{2} b_{i j}\right| \leq\left|z_{j}^{2} f_{i j}\right|
$$

for some real constants $f_{i j}$. Note that $f_{i j}=0$ if $i \neq j$.

Recalling Equation 21, let $A, B, C$, and $E$ be matrices such that $A(i, j)=a_{i j}, B(i, j)=b_{i j}, C(i, j)=$ $c_{i j}$, and $E(i, j)=e_{i j}+f_{i j}, i=j, i, j=1, \ldots, n$, and $A(i, j)=a_{i j} / 2, B(i, j)=b_{i j} / 2, C(i, j)=c_{i j} / 2$, and $E(i, j)=e_{i j} / 2+f_{i j} / 2, i \neq j, i, j=1, \ldots, n$. Then, a bound for $m(z)$ can be written in matrix form as $|m(z)|=\left|z^{T} M z\right|$, where

$$
M=\left[\begin{array}{cc}
0 & \frac{A+B \sqrt{\omega_{i}}}{2} \\
\frac{A+B \sqrt{\omega_{i}}}{2} & \frac{C+E \sqrt{\omega_{i}}}{2}
\end{array}\right]
$$

Using this bound on $m(z)$ in Equation 23 gives

$$
\begin{aligned}
\dot{V} \leq & -\omega_{i} z_{1}^{T} P_{12} \Omega z_{1}-\omega_{i} z_{2}^{T}\left(P_{22}\right) z_{2} \\
& +z_{2}^{T}\left(P_{12}\right) z_{2}+\left|z^{T} M z\right| .
\end{aligned}
$$

The matrices $P_{12}, P_{22}$ and $\Omega$ are positive definite and diagonal by construction. With the substitutions

$$
Q_{1}=\omega_{i}\left[\begin{array}{cc}
P_{12} \Omega & 0 \\
0 & P_{22}
\end{array}\right]
$$

and

$$
Q_{2}=\left[|M|+\left(\begin{array}{cc}
0 & 0 \\
0 & P_{12}
\end{array}\right)\right],
$$

Equation 30 becomes :

$$
\dot{V} \leq-|z|^{T}\left(Q_{1}-Q_{2}\right)|z| .
$$

From Equation 31, it is clear that $\dot{V}$ is negative definite if $Q=Q_{1}-Q_{2}$ is positive definite.

The matrix $Q$ is positive definite if all of its leading principal minors are positive definite. The matrix $Q$ is composed of terms that are constant, terms that are linear in $\omega_{i}$, and terms that are proportional to $\sqrt{\omega_{i}}$. Each diagonal element of $Q$ contains one of these linear terms, and all of them are on the diagonal. All of the terms which are linear in $\omega_{i}$ come from $Q_{1}$ and have positive coefficients. Therefore, the $n$ 'th principal minor has a determinant of the form $\kappa \omega_{i}^{n}+p(z)$, where $\kappa$ is a positive constant and $p(z)$ is composed of terms of order less than or equal to $n-\frac{1}{2}$ in $\omega_{i}$. Therefore, every leading principal minor of $Q$ will be positive definite for a large enough $\omega_{i}=\omega_{i}^{*}$ and $Q$ will be positive definite in $D$.

Since $Q$ is positive definite in $D, Q$ is positive definite in $S_{0}$, a subset of $\mathcal{D}$, and $\dot{V}$ is negative definite in $S_{0}$ and the system trajectory will never leave $S_{0}$. Therefore, the system (12) is exponentially stable about the origin and the original system $(1,2)$ is exponentially stable about $x=x_{c}$. 


\section{SINGULAR PERTURBATION ANAIYSIS}

Singular perturbation theory can also be applied to Equation 12 to analyze the stability of the system. Using standard singular perturbation theory results, such as those contained in [2], it is relatively simple to show that the origin of the system defined by Equation 12 is exponentially stable for large enough inner-loop gain $\omega_{i}^{*}$. However, this is a local result and does not guarantee any particular domain of attraction, as is found with the method used here.

\section{CONCLUSIONS}

In this paper, the stability of a nonlinear system of a certain form with a two time-scale dynamic inversion controller was studied. The closed-loop system is first converted into a form suitable for analysis. Then, under reasonable assumptions, the system is shown to be exponentially stable about constant commanded values of the slow states for a sufficiently large gain in the inner-loop dynamic inversion controller. The Lyapunov function used in the proof enables the calculation of a sufficient gain to guarantee asymptotic stability as well as a domain of attraction around the equilibrium at the commanded values.

\section{References}

[1] W, Hahn, "Stability of Motion," Springer-Verlag New York Inc. 1967, pp 102-115, 271-278.

[2] H. K. Khalil, "Nonlinear Systems," Macmillan Publishing Company, 1992, pp. 97-122, 136-151.

[3] P. V. Kokotovic, H. K. Khalil, editors, "Singular Perturbations in Systems and Control," IEEE Press, New York, 1986.

[4] P. V. Kokotovic, H. K. Khalil, J. O'Reilly, "Singular Perturbation Methods in Control: Analysis and Design" Academic Press, New York, 1986.

[5] M.B. McFarland and C.N. D'Souza, "Missile Flight Control With Dynamic Inversion and Structured Singular Value Synthesis," Proceedings of the AIAA Guidance, Navigation, and Control Conference, Scottsdale, AZ, 1994, pp. $544-550$.
[6] D. S. Naidu, A. J. Calise, "Singular Perturbations and Time Scales in Guidance, Navigation, and Control of Aerospace Systems: Survey," Proceedings of the AIAA Guidance, Navigation, and Control Conference, Baltimore, MD, 1995, pp. 1338-1362.

[7] C. Schumacher, "Tactical Missile Autopilots: Gain-Scheduled $\mathcal{H}_{\infty}$ Control and Dynamic Inversion," Ph.D. Thesis, Univ. of Michigan, Ann Arbor, MII, 1997.

[8] C. Schumacher, P. P. Khargonekar, "A Comparison of Missile Autopilot Designs Using $\mathcal{H}_{\infty}$ Control with Gain Scheduling and Nonlinear Dynamic Inversion" Proceedings of the American Controls Conference, Albuquerque, NM, 1997, pp. 2759-2763.

[9] C. Schumacher, P. P. Khargonekar, "Missile Autopilot Designs Using $\mathcal{H}_{\infty}$ Control with Gain Scheduling and Nonlinear Dynamic Inversion," Journal of Guidance, Control, and Dynamics, vol. 21 , no. 2 , pp. 234-243, 1998.

[10] C. Schumacher, P. P. Khargonekar, "Stability Analysis of a Missile Control System with a Dynamic Inversion Controller," Journal of Guidance, Control, and Dynamics, vol. 21, no. 3, pp. 508-515, 1998.

[11] S.A. Snell, D.F. Enns, and W.L. Garrard, "Nonlinear Inversion Flight Control for a Supermaneuverable Aircraft," Journal of Guidance, Control, and Dynamics, vol. 15, no. 4, pp. $976-$ 984, 1992. 